\title{
Fibrinogen/LDL apheresis is a promising rescue therapy for sudden sensorineural hearing loss
}

\author{
Martin Canis · Franz Heigl • Markus Suckfuell
}

Published online: 28 February 2012

(C) The Author(s) 2012. This article is published with open access at Springerlink.com

\begin{abstract}
Background Fibrinogen/LDL apheresis has been proven to be effective in treatment of sudden sensorineural hearing loss (SSNH). This study is aimed to investigate if reduction of fibrinogen and serum LDL is also effective in patients with SSNH non-responding toward treatment with corticosteroids and plasmaexpanders.

Methods Remission rates of 217 patients suffering from SSHL were investigated after treatment with apheresis. All patients were non-responders after other therapies such as high doses of steroids or plasmaexpanders.

Results Single apheresis resulted in complete or partial remissions in $61 \%$ of patients when given after other unsuccessful conducted therapies such as corticosteroids and plasmaexpanders.

Conclusion Fibrinogen/LDL apheresis is a promising rescue therapy for sudden sensorineural hearing loss even after unsuccessful other therapies.
\end{abstract}

Keywords Fibrinogen/LDL apheresis ·

Sudden sensorineural hearing loss - Observational study ·

Remission rate

M. Suckfuell $(\bowtie)$

Department of Otorhinolaryngology,

Head and Neck Surgery, Martha Maria Hospital,

Wolfratshauserstraße 109, 81479 Munich, Germany

e-mail: markus.suckfuell@martha-maria.de

\section{Canis}

Department of Otorhinolaryngology, Head and Neck Surgery,

University of Goettingen, Goettingen, Germany

\section{F. Heigl}

Dres. Heigl, Hettich \& Partner,

Medizinisches Versorgungszentrum Kempten-Allgäu,

Kempten-Allgäu, Germany

\section{Introduction}

With an estimated incidence of approximately 290 new cases per 100,000 residents per year, hearing loss is one of the most frequent inner ear disorders in western countries [1]. Even though there is often at least partial remission, lasting hearing loss and tinnitus are problematic consequences in many cases [2]. Notwithstanding, the pathogenesis is still largely unknown even today and is subject to controversial discussion. The most frequently discussed causes of hearing loss, also due to the clinical signs with sudden, one-sided commencement and possible spontaneous remission within hours or days, include disturbances in the cochlear microcirculation. Animal studies revealed that cochlear microcirculation is highly subject to disturbances and even slight changes in the regional blood flow may cause functional changes of the organ of Corti [3]. However, a monocausal occurrence is rather unlikely, so that numerous other causalities such as viral infections, immune and autoimmune pathological processes, endolymphatic hydrops or a sum of these mechanisms are under discussion [4]. Clinical evidence shows a negative effect of hyperlipidemia on hearing function [5] since high serum LDL (low density lipoprotein) and low HDL (high density lipoprotein) are commonly accepted as vascular risk factors. Beyond from its well known part in development of atherosclerosis and increase of blood viscosity, cholesterol can impair cochlear microcirculation by diminishing the release of the potent vasodilator nitric oxide (NO) from endothelial cells [6]. A second possible mechanism of hearing impairment by high serum cholesterol is a direct action at the outer hair cell (OHC) membrane. Isolated OHCs show diminished motility when incubated with a cholesterol enriched medium, probably due to increased stiffness caused by integration of cholesterol molecules into the lateral wall membrane [7]. 
Since fibrinogen is a large glycoprotein that defines rheological properties of whole blood by increasing plasma viscosity and inducing aggregation of erythrocytes, thrombocytes, and leucocytes, it is also thought to be a risk factor for inner ear disorders by reducing cochlear blood flow [8, 9]. Acutely lowering serum LDL and fibrinogen by means of LDL and fibrinogen apheresis thus increases cochlear blood flow without additional hemodilution and has been proven to be effective in treatment of idiopathic sudden sensorineural hearing loss. Interestingly, a minor $(1.8 \mathrm{~dB})$ but significant improvement of hearing threshold at the contralateral, healthy ear was also observed in the study [8]. Within the last years, the apheresis was used as therapeutic option in treatment of sudden sensorineural hearing loss in particular if standard treatment with steroids or hemodilution were ineffective. Because of the relevance for these patients, we aimed to assess whether lowering serum fibrinogen and LDL levels by fibrinogen/LDL apheresis may be an effective treatment for sudden sensorineural hearing loss in patients who are nonresponding toward other treatment options such as corticosteroids and plasmaexpanders.

\section{Patients and methods}

A retrospective study was performed to investigate remission rates in patients with SSHL after treatment with single fibrinogen/LDL apheresis. In particular, we wanted to answer the question if a single fibrinogen/LDL apheresis is an option in treatment of SSHL even if other therapies such as high doses of steroids or plasmaexpanders have been given unsuccessfully. We further wanted to compare the remission rates of different frequencies of hearing loss and different intervals between onset of SSHL and apheresis. As main outcome measure, we examined the differences of pure tone threshold before and after 2 weeks of treatment. Different frequencies of hearing loss were distinguished by means of pure-tone threshold: high-, low-, middle-frequency, pancochlear, deafness. Concerning the interval between onset of SSHL and apheresis, we defined the following groups - interval up to 14 days; interval between 14 and 42 days; interval between 42 and 90 days. Remission rates were grouped concerning pure tone threshold and patient reports as follows - complete remission; partial remission; no change; worsening.

For this purpose, the patients were treated within the Medical Care Unit, Kempten-Allgäu and the University of Munich, Campus Großhadern between January 2004 and June 2009. The study was done in accordance with the declaration of Helsinki. All patients provided written informed consent. An independent office (Medizinisches Wirtschaftsinstitut, Munich, Germany) monitored and analyzed the data. The patients, from 18-80 years of age suffering from acute SSHL which previously had been treated unsuc- cessfully using therapies such as high doses of steroids or plasmaexpanders, were included.

Audiometric testing such as pure-tone audiometry (frequencies 125, 250, 500, 1000, 2000, 4000, and $8000 \mathrm{~Hz}$ ), tympanometry and stapedius reflex measurements was performed before treatment and 2 weeks after therapy in accordance with ISO 7029. All laboratory tests were done immediately before and after treatment at the same laboratory using standard methods. We measured the concentrations of total cholesterol, HDL cholesterol, LDL cholesterol, triglycerides and lipoprotein(a), sodium, potassium, calcium, urea, glucose, creatinine, total protein, bilirubin, uric acid, albumin, high sensitivity C-reactive protein, alanine transaminase, and aspartate transaminase. We also assessed leucocytes, erythrocytes, thrombocytes, hemoglobin, packed-cell volume, mean corpuscular volume, mean corpuscular hemoglobin, mean corpuscular hemoglobin concentration, the concentration of fibrinogen, the international normalization ratio, and the partial thrombin time. The patients having a clotting disorder, malignant disease, hepatitis B or C, HIV-1, HIV-2, or dementia were excluded. The procedure of fibrinogen/LDL apheresis has been described previously as H.E.L.P.-apheresis (Heparin induced LDLPrecipitation). We treated $3 \mathrm{~L}$ plasma within $2 \mathrm{~h}$ (Plasmat ${ }^{\mathbb{B}}$ Futura, B Braun Medizintechnologie $\mathrm{GmbH}$, Melsungen, Germany) on an outpatient basis. This procedure reduced plasma cholesterol, LDL, fibrinogen, and lipoprotein(a) by more than $50 \%$. The data were collected, checked for entry errors, validated, and corrected. The statistical evaluation is descriptive and was performed with SAS (Version 9.1) for Windows and the software SPSS (Version 12.0). The Wilcoxon signed-rank test was used for comparison between the two groups. A $p$ value of $\alpha \leq 0.05$ was judged significant.

\section{Results}

Data were presented at the Symposium on Therapeutic Apheresis Bad Oeynhausen October 2012 and were already published elsewhere [10]. Table 1 shows the demographic

Table 1 The composition as well as the cardiovascular risk factors of all patients. Values are given as numbers percent or mean $\pm \mathrm{sd}[10]$

\begin{tabular}{ll} 
Patients characteristics & \\
Gender & $\mathrm{n}=144(66 \%)$ \\
Male & $\mathrm{n}=73(33 \%)$ \\
Female & $56.89 \pm 14.00$ \\
Age (years) & $\mathrm{n}=46(21 \%)$ \\
Hypertension & $\mathrm{n}=32(12 \%)$ \\
Nicotine consumption & $\mathrm{n}=69(32 \%)$ \\
Hyperlipidemia & $\mathrm{n}=6(3 \%)$ \\
Diabetes mellitus & \\
\hline
\end{tabular}


Table 2 Laboratory data [10]

\begin{tabular}{llll} 
& $\begin{array}{l}\text { Before } \\
\text { apheresis }\end{array}$ & After apheresis & $\begin{array}{l}\text { Reduction } \\
\text { rate }(\%)\end{array}$ \\
\hline $\begin{array}{l}\text { Total cholesterol } \\
(\mathrm{mg} / \mathrm{dl})\end{array}$ & $218.45 \pm 46.02$ & $115.27 \pm 25.28^{* *}$ & 47.2 \\
$\begin{array}{l}\text { HDL cholesterol } \\
(\mathrm{mg} / \mathrm{dl})\end{array}$ & $57.65 \pm 14.38$ & $46.62 \pm 10.56^{* *}$ & 19.1 \\
$\begin{array}{l}\text { LDL cholesterol } \\
(\mathrm{mg} / \mathrm{dl})\end{array}$ & $127.17 \pm 41.75$ & $55.50 \pm 22.91^{* *}$ & 56.4 \\
$\begin{array}{l}\text { Triglycerides } \\
(\mathrm{mg} / \mathrm{dl})\end{array}$ & $195.47 \pm 122.41$ & $85.74 \pm 22,64^{* *}$ & 56.1 \\
$\begin{array}{l}\text { Lipoprotein(a) } \\
(\mathrm{mg} / \mathrm{dl})\end{array}$ & $30.61 \pm 28.37$ & $11.60 \pm 9.53^{* *}$ & 62.1 \\
$\begin{array}{l}\text { Fibrinogen } \\
(\mathrm{mg} / \mathrm{dl})\end{array}$ & $308.70 \pm 90.95$ & $118.58 \pm 54.51^{* *}$ & 61.6 \\
\hline
\end{tabular}

$* * p<0.001$

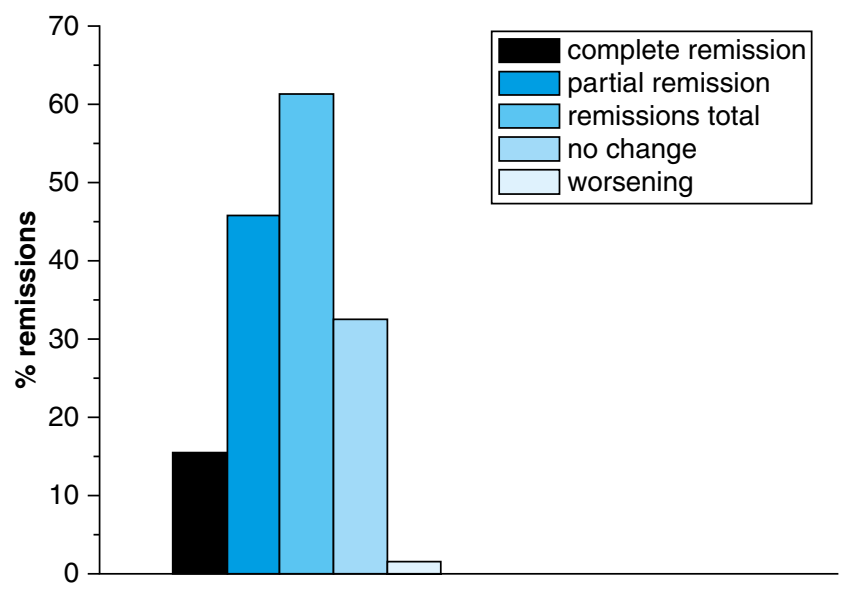

Fig. 1 The remission rates of all patients in percent [10]

data as well as the cardiocirculatory risk factors. Lipids and fibrinogen before and after treatment are given in Table 2. Laboratory tests revealed significant $(p<0.001)$ decreases of fibrinogen and plasma lipids by fibrinogen/LDL apheresis. Regarding all patients and frequencies, complete remissions were observed in $15 \%$ of the cases, whereas partial remissions were seen in $46 \%$. In $33 \%$ of the patients, we observed no change while $2 \%$ worsened (Fig. 1). Data were missing in $4 \%$ of the cases.

Figure 2 shows the remission rates divided into different frequencies of hearing loss. When we grouped complete and partial remissions (remissions total), we observed remission rates of $61 \%$ in the high frequency group, $74 \%$ in low frequencies, $40 \%$ in middle frequencies, $66 \%$ in the pancochlear SSHL group, and $40 \%$ in deaf patients. Figure 3 gives evaluated hearing thresholds (frequencies 125, 250, $500,1000,2000,4000$, and $8000 \mathrm{~Hz}$ ) of all patients. After a single fibrinogen/LDL apheresis, a significant $(p<0.001)$ increase in the mean threshold in all frequencies was obser-

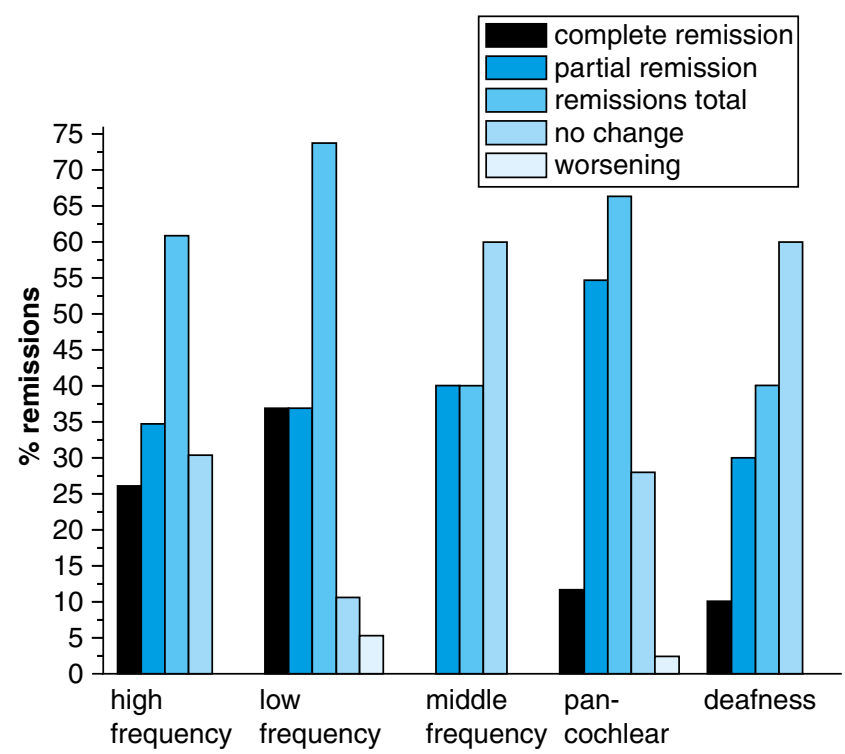

Fig. 2 The remission rates divided into different frequencies of hearing loss [10]

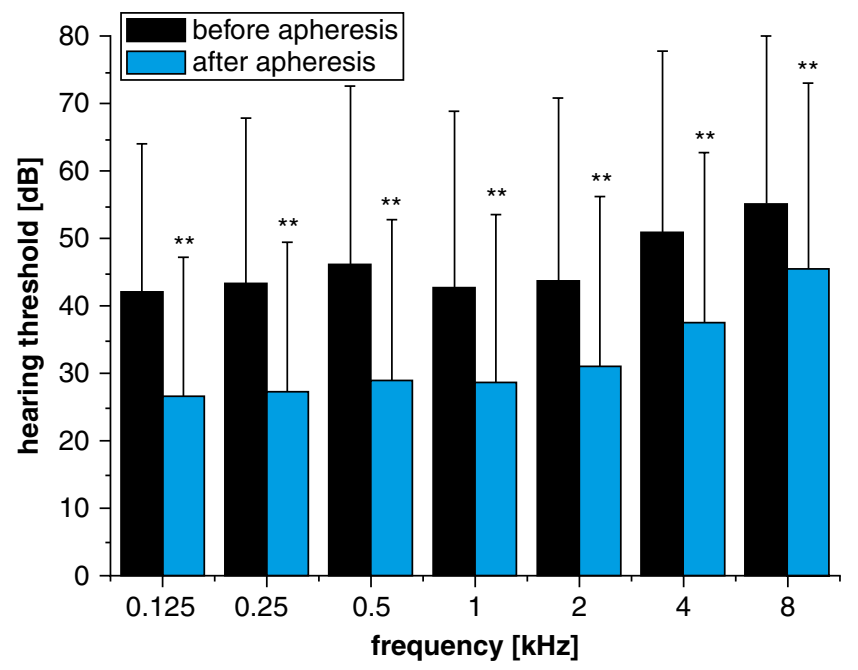

Fig. 3 The hearing thresholds before and after fibrinogen/LDL apheresis in percent [10]

vable. The mean increase over all the patients and frequencies was $14.9 \pm 2.7 \mathrm{~dB}$.

Figure 4 gives remission rates depending on the interval between onset of SSHL and fibrinogen/LDL apheresis. The mean interval was $27.4 \pm 34.7$ days. The number of complete remissions decreased from $32 \%$ within 14 days to $15 \%$ within 42 days. After 90 days, no complete remissions were observable anymore. Also the number of remissions total (complete + partial remissions) decreased from $70 \%$ after 2 weeks between onset of SSHL and apheresis to 63 and $21 \%$ for an interval of 6 weeks and 3 months respectively. 


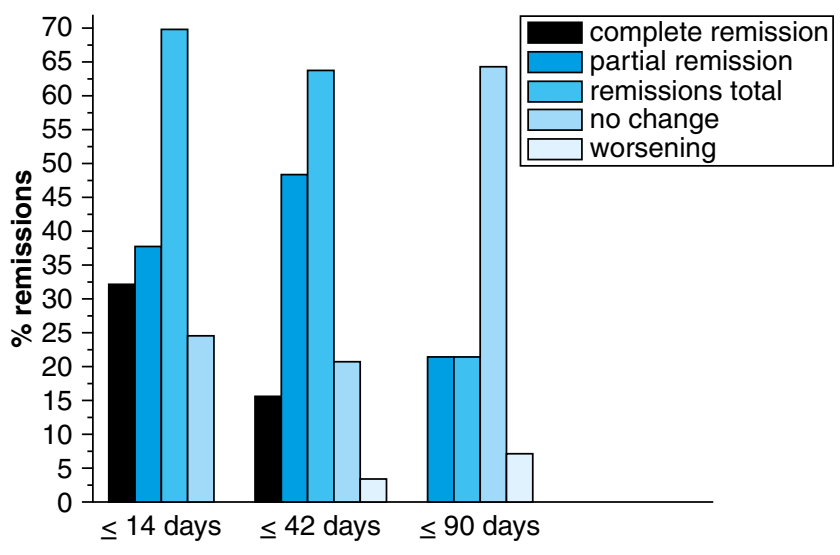

Fig. 4 The remission rates depending on the interval between onset of SSHL and apheresis [10]

\section{Discussion}

Little is known about the pathogenesis of idiopathic hearing loss; nonetheless, disturbances in the cochlear microcirculation are among the most commonly discussed causes. In a significant number of patients, the clinical signs of hearing loss are similar to those of other vascular disorders such as stroke or myocardial infarction. Blood supply of the inner ear is provided by the labyrinthic artery which is a functional end artery, and thus, shunting from the periphery cannot compensate for disturbances in regional blood flow. Furthermore, the labyrinthic artery supplies the vestibulocochlear artery and the spiralis modiolic artery, which supply the cochlea and the vestibular organ. More than $30 \%$ of the patients also have vestibular symptoms supporting the hypothesis that SSHL may be of vascular origin. However, the association of hearing loss and vascular risk factors is still subject to controversial discussion. Nonetheless, numerous therapeutic modalities were described and implemented under the hypothesis of vascular pathology of hearing loss in recent years. It was thus far not possible to prove the success of these therapies, since there are only very few clinical data which represent the remission rates of various types of hearing loss and therapeutic modalities while taking the time-related therapeutic window into account. A therapeutic option commonly used in Europe consists of glucosteroids and hypervolemic hemodilution by means of plasma replacement products.

The effect of corticosteroids is based on an antiinflammatory and swelling-reducing effect with the assumption that an inflammation or immune system modulated reaction on the inner ear may be causative for the development of hearing loss. The effect which reduces swelling as well as the increased response to catecholamines is also intended to increase cochlear blood flow. As for the rheological infusion therapy, there are also few placebo-controlled clinical studies about the effectiveness of steroid therapy in the tre- atment of idiopathic hearing loss. Existing studies furthermore show nonuniform results, so that a metaanalysis which was published in 2006 [11] herein also shows the unproven effect of cortisone.

The use of plasma expanders increases the circulating blood volume and simultaneously reduces the relative share of the cellular part of the blood. The consequence is an increased heart time volume with simultaneously improved flow attributes of the whole blood. The objective of therapeutic improvement of the blood's flowing behavior is to improve the microcirculation, since this is required for optimal supply of oxygen and energy carriers to the inner ear as well as removal of metabolic end products. Hydroxyethyl starch solutions find particular use in clinical practice. Aside from the effect of hemodilution, HES also brings about a reduction of inflammation signs and endothelial cell activation [12]. Overall, no study which contained at least 50 rheologically treated patients showed a hearing gain or a higher remission rate as compared to the control group which was treated with placebo. As a side effect of therapy with HES, a dosage dependent, long lasting, but reversible itching sensation has been described. With high dosing of HES, cumulative embedment of hydroxyethyl starch of more than $180 \mathrm{~g}$ into the reticuloendothelial system is discussed as causative.

Fibrinogen/LDL apheresis is an established procedure to acutely reduce the concentration of serum LDL and lipoprotein(a) to $60 \%$ and that of fibrinogen to $65 \%$ of the original values. Reduction of plasma fibrinogen improves the blood's rheological properties and results in a significant reduction of plasma viscosity, an improvement of endothelial functions in coronary and peripheral arteries [13] as shown by positron emission tomography [14] and an increase in tissue oxygenation as measured in muscle with polarographic needle methods [15, 16]. Furthermore, the reduction of serum cholesterol increases the release of nitric oxide and could reestablish autoregulation of cochlear blood flow. Hence, the reduction of plasma LDL cholesterol by apheresis treatment increases cochlear blood flow in animals and has been used in patients with SSHL. In a prospective study, Shiraishi et al. [17] showed a better hearing recovery in patients by lowering fibrinogen due to the venom enzyme batroxobin in comparison to steroids. Acutely lowering the fibrinogen and serum LDL by means of fibrinogen/LDL apheresis has also been proven effective in a clinical trial. In a prospective randomized clinical study, Suckfuell et al. [8] showed that pure-tone thresholds in patients with sudden hearing loss improved more, but not significantly attributable to apheresis compared to plasmaexpander and prednisolone treatment. Interestingly, also a minor but significant elevation of the hearing threshold of the healthy contralateral ear was observed in this study. However, this study did not evaluate rates of remission in regard to different frequencies and interval between first symptoms and apheresis, since patients were 
included within 1 week after SSHL only. Another question was if apheresis is helpful after unsuccessfully conducted therapies such as high doses of steroids or plasmaexpanders.

To this aim, we retrospectively investigated the outcome of a single fibrinogen/LDL apheresis as treatment of SSHL after not responding toward other therapies such as corticosteroids and plasmaexpanders. If regarding all patients and frequencies, complete remissions were observed in $15 \%$, partial remissions were seen in $46 \%$. These data are in line with a previously reported cohort of 152 patients [18], where remission rates of $11 \%$ (complete remission) and $43 \%$ (partial remissions) have been found. The better results of the present study may be explained by the fact that fibrinogen was significantly $(p<0.01)$ higher $(308.7 \pm 90.95 \mathrm{mg} / \mathrm{dl}$ vs. $282.23 \pm 75.22 \mathrm{mg} / \mathrm{dl}$ ). This observation is in line with results of Suckfuell et al. [8]. In this study, the outcome of fibrinogen/LDL apheresis was significantly better in patients with higher LDL and fibrinogen concentrations. Furthermore, the interval between onset of SSHL and apheresis was slightly but not significantly shorter $(27 \pm 35$ days vs. $31 \pm 41$ days). Complete remissions decreased from $32 \%$ within 14 days to $15 \%$ within 42 days. After 42 days, no complete remissions were observable anymore. These data approve previous results [18] showing a rapid decrease in remissions after latency of apheresis of more than 6 weeks. Notwithstanding, spontaneous remission of hearing is estimated to about $65 \%$ [19]. However, the high rate of mostly part remissions usually takes place within the first 2 weeks. This fact corroborates the efficacy of fibrinogen/LDL apheresis in treatment of SSHL as successful second-line therapy even at times when spontaneous remissions are not expected anymore [10].

\section{Conclusion}

After not responding toward other therapeutic options such as corticosteroids and plasmaexpanders, the 217 patients presenting with SSHL were treated with single apheresis. Complete remissions were reported in $15 \%$, partial remissions were seen in $46 \%$. The therapeutic window is approximately 6 weeks. High remission rates corroborate the effects of fibrinogen/LDL apheresis in treatment of SSHL.

Acknowledgments This article is part of a supplement sponsored by an unrestricted educational grant from B. Braun and Fresenius Medical Care.

Disclosures M. Suckfuell has received consulting fees from B. Braun Medizintechnologie.

Conflict of interest The authors declare that there is no actual or potential conflict of interest in relation to this article.
Open Access This article is distributed under the terms of the Creative Commons Attribution License which permits any use, distribution, and reproduction in any medium, provided the original author(s) and the source are credited

\section{References}

1. Olzowy B, Osterkorn D, Suckfull M (2005) The incidence of sudden hearing loss is greater than previously assumed. MMW Fortschr Med 147:37-38

2. Canis M, Osterkorn D, Osterkorn K et al (2009) A retrospective assessment of 741 patients with sudden hearing loss. Open Otorhinolaryngol J 3:5-10

3. Miller JM, Dengerink H (1988) Control of inner ear blood flow. Am J Otolaryngol 9:302-316

4. Byl FM Jr (1984) Sudden hearing loss: Eight years' experience and suggested prognostic table. Laryngoscope 94:647-661

5. Sidman JD, Prazma J, Pulver SH et al (1988) Cochlea and heart as end-organs in small vessel disease. Ann Otol Rhinol Laryngol 97:9-13

6. Feron O, Dessy C, Desager JP et al (2001) Hydroxy-methylglutaryl-coenzyme A reductase inhibition promotes endothelial nitric oxide synthase activation through a decrease in caveolin abundance. Circulation 103:113-118

7. Nguyen TV, Brownell WE (1998) Contribution of membrane cholesterol to outer hair cell lateral wall stiffness. Otolaryngol Head Neck Surg 119:14-20

8. Suckfull M (2002) Fibrinogen and LDL apheresis in treatment of sudden hearing loss: A randomised multicenter trial. Lancet 360:1811-1817

9. Suzuki K, Kaneko M, Murai K (2000) Influence of serum lipids on auditory function. Laryngoscope 110:1736-1738

10. Heigl F, Hettich R, Suckfuell M et al (2009) Fibrinogen/LDL apheresis as successful second-line treatment of sudden hearing loss: A retrospective study on 217 patients. Atheroscler Suppl 10:95-101

11. Wei BP, Mubiru S, O’Leary S (2006) Steroids for idiopathic sudden sensorineural hearing loss. Cochrane Database Syst Rev 1:CD003998

12. Boldt J, Ducke M, Kumle B et al (2004) Influence of different volume replacement strategies on inflammation and endothelial activation in the elderly undergoing major abdominal surgery. Intensive Care Med 30:416-422

13. Pfefferkorn TK, Knuppel HP, Jaeger BR et al (1999) Increased cerebral $\mathrm{CO}(2)$ reactivity after heparin-mediated extracorporal LDL precipitation (HELP) in patients with coronary heart disease and hyperlipidemia. Stroke 30:1802-1806

14. Mellwig KP, van Buuren F, Schmidt HK et al (2006) Improved coronary vasodilatatory capacity by H.E.L.P. apheresis: Comparing initial and chronic treatment. Ther Apher Dial 10:510-517

15. Kojima Y, Ito S, Furuya N (2001) Hearing improvement after therapy for hyperlipidemia in patients with chronic-phase sudden deafness. Ann Otol Rhinol Laryngol 110:105-108

16. Seidel D (1996) H.E.L.P. apheresis therapy in the treatment of severe hypercholesterolemia: 10 years of clinical experience. Int J Artif Organs 20:303-310

17. Shiraishi T, Kubo T, Okumura S et al (1993) Hearing recovery in sudden deafness patients using a modified defibrinogenation therapy. Acta Otolaryngol Suppl 501:46-50

18. Canis M, Heigl F, Hettich R et al (2008) Fibrinogen/LDL apheresis for treatment of sudden hearing loss: An observational study on 152 patients. HNO 56:961-966

19. Mattox DE, Simmons FB (1977) Natural history of sudden sensorineural hearing loss. Ann Otol Rhinol Laryngol 86:463-480 